\title{
Metadados para a descrição de recursos de informação eletrônica: utilização do padrão Dublin Core
}

Marcia Izabel Fugisawa Souza

Bacharel em biblioteconomia, UEL, Londrina, PR, 1978.

Mestre em biblioteconomia, Puccamp, Campinas, SP, 1992. e-mail:marcia@cnptia.embrapa.br

Laurimar Gonçalves Vendrusculo

Bacharel em engenharia elétrica, UFMG, Belo Horizonte,

MG, 1988. Mestranda em engenharia agrícola,

Unicamp-Feagri.

e-mail: laurimar@cnptia.embrapa.br

Geane Cristina Melo

Bacharel em publicidade e propaganda, Unip, Campinas, SP. e-mail:mageval@bestway.com.br

\section{Resumo}

Este artigo aborda a necessidade de adoção de padrões de descrição de recursos de informação eletrônica, particularmente, no âmbito da Embrapa Informática Agropecuária. O Rural Mídia foi desenvolvido utilizando o modelo Dublin Core (DC) para descrição de seu acervo, acrescido de pequenas adaptações introduzidas diante da necessidade de adequar-se a especificidades meramente institucionais. Este modelo de metadados baseado no Dublin Core, adaptado para o Banco de Imagem, possui características que endossam a sua adoção, como a simplicidade na descrição dos recursos, entendimento semântico universal (dos elementos), escopo internacional e extensibilidade (o que permite sua adaptação às necessidades adicionais de descrição).

\section{Palavras-chave}

Metadados; Dublin Core; Informação eletrônica; Recursos de informação; Catalogação de recursos eletrônicos.

\section{Metadata for description of electronic information resources: the use of Dublin Core}

\begin{abstract}
The objective of this article is the need of adoption of eletronic information resources patterns, particularly, in the Embrapa Agricultural Informatics area. The Rural Midia was developed using Dublin Core model for description of its collection, increased of small adaptations introduced due to the need of institutional adjusts. This metadados model based on Dublin Core, adapted for the Image Bank, has characteristics that endorse its adoption, as the simplicity in the resources description, universal semantic understanding (of the elements), international uses and extensibility (what allows its adaptation to the additional description needs).
\end{abstract}

\section{Keywords}

Metadata; Dublin Core; Electronic information; Electronic resources cataloguing; Information resources.

Artigo aceito para publicação em 09/05/2000

\section{DESCRIÇÃO DE RECURSOS DE INFORMAÇÃO - PADRÃ̃O DUBLIN CORE}

Com a expansão da Internet e o conseqüente desenvolvimento da tecnologia de redes eletrônicas, vêm se intensificando o fenômeno da explosão de documentos eletrônicos, ocasionando o aumento, em progressão geométrica, do volume de informações disponíveis. Diante dessa realidade, torna-se imprescindível o desenvolvimento de padrões que visem à descrição exata dos recursos de informação. Nesse sentido, várias iniciativas estão sendo conduzidas com o propósito de discutir a questão e propor padrões de descrição de recursos de informação, como é o caso do Dublin Core Metadata Initiative, em cujo site estão disponíveis as mais recentes contribuições apresentadas pelo Working Group aos membros do DC.

Dublin Core pode ser definido como sendo o conjunto de elementos de metadados planejado para facilitar a descrição de recursos eletrônicos. Metadado significa dado sobre o dado. É a catalogação do dado ou descrição do recurso eletrônico. A expectativa é que autores ou websiters sem conhecimento de catalogação sejam capazes de usar o Dublin Core para descrição de recursos eletrônicos, tornando suas coleções mais visíveis pelos engenhos de busca e sistemas de recuperação. Na maioria dos casos, o conjunto de descritores do Dublin Core é embutido no próprio documento descrito (HTML, XML - Extensible Markup Language e outros), ou, dependendo do recurso, a meta-informação encontra-se separada do recurso catalogado.

O conjunto de metadados descrito pelo DC é composto de 15 elementos, os quais poderiam ser descritos como o mais baixo denominador comum para descrição de recurso (equivalente a uma ficha catalográfica). Entretanto, o Dublin Core não tem a intenção de substituir modelos mais ricos como o código AACR2/MARC, mas apenas fornecer um conjunto básico de elementos de descrição que podem ser usados por catalogadores ou nãocatalogadores para simples descrição de recursos de informação (Weibel, 1997). 
As principais características do padrão DC são a simplicidade na descrição dos recursos, entendimento semântico universal (dos elementos), escopo internacional e extensibilidade (o que permite sua adaptação às necessidades adicionais de descrição).

\section{A EXPERIÊNCIA DA EMBRAPA INFORMÁTICA AGROPECUÁRIA: BANCO DE IMAGEM RURAL MÍDIA (BI-RM)}

Instituições envolvidas na organização da informação em ambiente web, como a construção de bibliotecas digitais, bases de dados, portais e sites, entre outros serviços, estão se deparando com a necessidade de implementar padrões de descrição de seus recursos eletrônicos. No âmbito da Embrapa, particularmente em unidades de pesquisa envolvidas com a geração, difusão e aplicação de tecnologia de informação e de comunicação, registra-se também a crescente necessidade de estabelecimento de padrões de descrição de conteúdos de informação. Um exemplo dessa necessidade pode ser ilustrado pela experiência recente com o desenvolvimento do Banco de Imagem - Rural Mídia, criado pela da Embrapa Informática Agropecuária, para armazenar e oferecer acesso ao seu acervo de fotos, ícones e gravuras. A criação do Banco de Imagem consistiu em uma oportunidade pioneira para a discussão e o estabelecimento de uma metodologia de tratamento de quaisquer recursos eletrônicos de informação, sejam estes recursos visuais ou outros tipos de documentos eletrônicos em seus variados formatos, com vistas a assegurar um padrão mínimo de descrição. $\mathrm{O}$ padrão de descrição de recursos de informação adotado pelo Banco de Imagem e proposto pela Embrapa Informática Agropecuária para descrever qualquer outro tipo de recurso na Embrapa é o Dublin Core, acrescido de pequenas adaptações introduzidas diante da necessidade de adequar-se meramente a especificidades institucionais.

A aplicação BI-RM foi desenvolvida para o ambiente da rede Internet com enfoque em recursos visuais de informação. A estratégia para implementar a estrutura do DC foi desenvolvida por meio do sistema gerenciador de base de dados (SGBD) MS-ACCESS, o qual armazenou os registros relativos à catalogação de cada imagem.

Este documento apresenta uma versão adaptada do capítulo "The core elements", do texto "A user guide for simple Dublin Core: draft version 5.1 (Dublin ..., 1999). Essa adaptação é baseada em necessidades detectadas por profissionais envolvidos, especificamente, com a descrição de conteúdos de informação eletrônica. Ao conjunto de elementos do DC, foram introduzidos três novos: Categoria, Acesso e Contato.
Elemento Categoria - Embora exista elemento equivalente no DC para Categoria, que é o Subject and Keywords, optou-se pelo desmembramento deste, por considerar que ambos, isoladamente, possuem conceitos e descrições diferenciados, apesar de estarem relacionados ao conteúdo.

Elemento Acesso - Este elemento tem a descrição equivalente à prevista no elemento Identificador de Recurso do DC; porém, apesar de mantido nessa adaptação, deve ser descrito como uma string ou número usado para identificar um recurso dentro da base de dados. Dessa forma, restou a necessidade de um elemento identificador do recurso, externamente, no qual podem ser incluídos URL, URN, ISBN e outros identificadores.

Elemento Contato - Elemento identificador da pessoa e/ ou instituição diretamente relacionada com o recurso descrito, com os quais se pode estabelecer contato para obter informações adicionais, aspectos estes que determinaram a sua inclusão como elemento de descrição de recurso de informação.

\section{ELEMENTOS DE METADADOS}

Estão apresentados, a seguir, os 18 elementos que compõem a versão adaptada do DC: Título; Autor ou Criador; Palavras-chave; Categoria; Descrição; Publicador; Colaborador; Data; Tipo; Formato; Acesso; Identificador de recurso; Fonte; Idioma; Relação; Cobertura; Direito autoral; Contato.

Esta seção apresenta a descrição de cada elemento do conjunto de metadados, seguida de orientação para auxiliar na criação do conteúdo do metadado e de exemplos. Os elementos estão apresentados pela ordem natural de seu desenvolvimento, embora seja possível exibi-los também por agrupamentos relacionados a conteúdo, propriedade intelectual e instanciação.

\begin{tabular}{lll}
\hline Conteúdo & $\begin{array}{l}\text { Propriedade } \\
\text { intelectual }\end{array}$ & Instanciação \\
\hline $\begin{array}{l}\text { Título } \\
\text { Palavra-chave }\end{array}$ & $\begin{array}{l}\text { Criador } \\
\text { Publicador/editor }\end{array}$ & $\begin{array}{l}\text { Data } \\
\text { Formato/ } \\
\text { tamanho } \\
\text { Identificador } \\
\text { Categoria }\end{array}$ \\
$\begin{array}{l}\text { Descrição } \\
\text { Tipo }\end{array}$ & Colaborador \\
Fonte & Direitos autorais \\
Contato & & Idioma \\
Cobertura & & Relação
\end{tabular}




\section{Título}

Descrição: O nome dado para o recurso

\section{Autor ou Criador}

A(s) pessoa(s) ou organização(ões) principal(is) responsável(is) pela criação do conteúdo intelectual do recurso. Ex.: autores, no caso de documentos escritos; artistas, fotógrafos ou ilustradores, no caso de recursos visuais.

\section{Palavras-chave}

A essência do contéudo do recurso. $\mathrm{O}$ assunto deve ser expressado por palavras-chave ou frase que descrevam o assunto ou conteúdo do recurso. $\mathrm{O}$ uso de vocabulários controlados é encorajado.

\section{Categoria}

Atribuição de categoria de assunto que expresse a essência do conteúdo do recurso. $\mathrm{O}$ uso de sistemas de classificação formais é encorajado, como o Agricola, Dewey Decimal Classification.

\section{Descrição}

Uma descrição textual do conteúdo do recurso, incluindo abstracts, no caso de documentos textuais, ou descrição de conteúdo, no caso de recursos visuais.

\section{Publicador}

A entidade responsável por tornar o recurso disponível na presente forma, tais como uma casa publicadora, um departamento de uma universidade, uma entidade.

\section{Colaborador}

Pessoa ou organização não especificada no elemento Criador que tenha dado contribuição intelectual significativa para o recurso, mas cuja contribuição é considerada secundária para a pessoa ou instituição especificada no elemento Criador, como, por exemplo, editor, tradutor, ilustrador.

\section{Data}

A data em que o recurso tornou-se disponível na presente forma.
Uma data associada com a criação ou disponibilidade do recurso. Tal data não deve ser confundida com o descrito no elemento Cobertura, o qual está associado ao conteúdo intelectual. Recomenda-se adotar a ISO 8601 (Formatos de datas e de tempo, W3C Technical Note http:// www.w3.org/TR/NOTE-datetime), que inclui (entre outros) datas nas formatos YYYY e YYYY-MM-DD. Neste esquema, a data 1999-11-07 corresponde a 7 de novembro de 1999.

\section{Tipo}

A categoria do recurso, como texto, imagem, som, dados, software, interativo, evento e objeto físico. Tipo de recurso deve ser selecionado da lista abaixo.

\section{Formato}

O formato do dado do recurso, usado para identificar o software e possivelmente o hardware que pode ser necessário à exibição ou operação do recurso. Formato deve ser selecionado de uma lista em desenvolvimento pelo Dublin Core Workshop Series.

\section{Acesso}

Identificador de recursos capturados da rede, como URLs e URNs (quando implementadas). Outros identificadores internacionalmente conhecidos, International Standard Book Number (ISBN), ou outros nomes formais também são candidatos a este elemento.

\section{Identificador de recurso}

String ou número usado para identificar um recurso, de forma única.

\section{Fonte}

Informação sobre um segundo recurso do qual o presente recurso é derivado. Embora seja recomendável que elementos contenham informação extraída do presente recurso apenas, o elemento Fonte pode conter uma data, criador, formato, identificador ou outro metadado de um segundo recurso quando este é considerado importante para a identificação do presente recurso. Uma prática recomendada é o uso do elemento Relação em seu lugar. Por exemplo, é possível usar uma data 1603 em Fonte em uma descrição de uma adaptação de 1996 feita para cinema de uma peça de Shakespeare, porém é preferível, ao invés, usar o elemento Relação "Baseado em", com uma referência para um recurso separado cuja descrição contém uma data de 1603. Fonte não é aplicável, se o recurso presente está em sua forma original. 


\section{Idioma}

O idioma do conteúdo intelectual do recurso.

\section{Relação}

Possibilita relacionamento com outros recursos. A especificação desse elemento visa a fornecer um meio de expressar relacionamentos entre recursos que têm relação formal com outros, mas que existem por si mesmos.

Ex.: imagens em um documento, capítulos em um livro, versão de 1990 de uma obra originalmente publicada em 1888 .

\section{Cobertura}

As características espaciais ou temporais do conteúdo intelectual do recurso. Cobertura espacial refere-se a uma região física (por exemplo, celestial); use coordenadas (por exemplo, longitude e latitude) ou nomes de lugares de uma lista controlada. Cobertura temporal refere-se sobre o que é o recurso, e não quando foi criado ou tornado disponível; use o mesmo formato de data/tempo [Data e Formatos de Tempo (baseado na ISO8601), W3C Technical Notes, http://www.w3.org/TR/NOTEdatetime] recomendado para o elemento Data, ou, para períodos de tempo, use lista controlada ou amplamente conhecida.

\section{Direito autoral}

Uma declaração de direito sobre a propriedade, um identificador que vincula a uma declaração de direito sobre a propriedade, ou um identificador que vincula a um serviço que fornece informação sobre o direito de propriedade do recurso.

\section{Contato}

Indicação para contato de nome e e-mail da pessoa ou instituição à qual o recurso está vinculado.

\section{O POTENCIAL DA METODOLOGIA DE DESCRIÇÃO DE METADADOS}

O Dublin Core é uma ferramenta que oferece ampla oportunidade de uso, para descrição de vários tipos de recursos, envolvendo os mais variados formatos de documentos. A Embrapa Informática Agropecuária está conduzindo projeto de pesquisa dedicado ao desenvolvimento de sites, os quais prevêem a utilização da mesma metodologia para o tratamento da informação a ser inserida em suas bases de dados. O emprego da estrutura de metadados do DC, até o momento, tem se mostrado satisfatório para a descrição de recursos de tipo texto, principalmente para documentos como home page e site, base de dados, imagem e eventos.

\section{APLICAÇÃO ON-LINE DO BANCO DE IMAGEM RURAL MÍDIA}

Desenvolveu-se a aplicação Web Rural Mídia, a qual emprega as tecnologias HTML, JAVA applets e a ferramenta CGI (Common Gateway Interface) Utilis 1.0* (figura 1). $\mathrm{O}$ acesso a mesma encontra-se no domínio (URL) http://wwwbases.cnptia.embrapa.br/RuralMidia. Este CGI genérico permite o desenvolvimento de sistemas para gerência de informações armazenadas em base de dados por meio da Web, com rapidez e eficiência. A metodologia usada para o desenvolvimento das aplicações de consulta às bases de dados é descrita por Moura \& Oliveira (1998).

\section{FIGURA 1}

\section{Modelo da aplicação Web do BI-RM.}

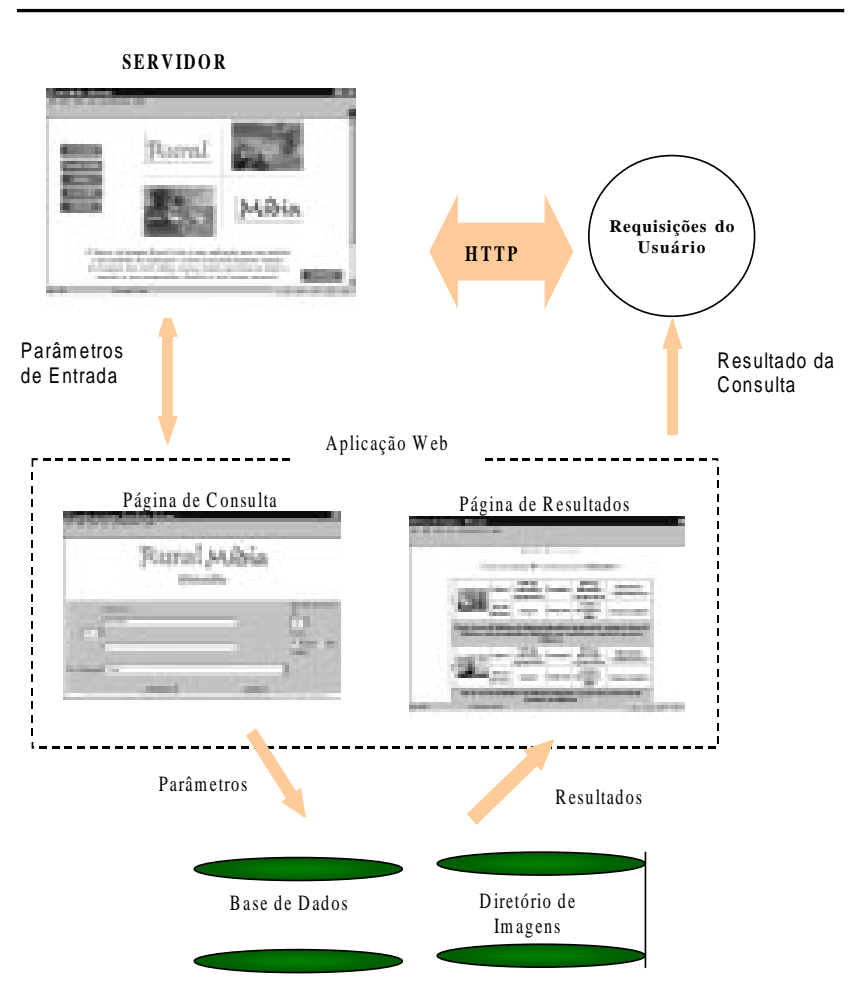

* Tecnologia desenvolvida pela Embrapa Informática Agropecuária, anteriormente denominada de mtform.cgi. 
O BI-RM foi modelado seguindo a estrutura de atributos do DC no SGBD MS-ACCESS. Em paralelo, a atividade de tratamento das imagens foi iniciada (aplicação de técnicas de processamento de imagens com vistas à sua adequação à Internet). Desta etapa, obtiveram-se diretórios de imagens em formatos .gif e .jpg , correspondentes ao tamanho natural e a uma versão reduzida das imagens.

Para consulta ao acervo de imagens, construiu-se uma aplicação Web utilizando o CGI Utilis, o qual permite a interação com o software MS-ACCESS e a associação da respectiva imagem no diretório apropriado.

O módulo de consulta do sistema Rural Mídia permite a entrada de até duas palavras-chave combinadas com os operadores and e or. Internamente, a aplicação utiliza esta entrada como parâmetros para formar uma consulta na linguagem Structured Query Language (SQL) e submetida ao SGBD. Esta consulta embutida, somada aos parâmetros de entrada, permite a recuperação da imagem e dos metadados. Resumindo, é criado, dinamicamente, segundo a solicitação do usuário, o metadocumento relativo ao recurso solicitado.

A escolha da abordagem utilizando banco de dados para o armazenamento dos metadados e diretório de imagens mostrou-se eficiente e rápida no processo de consulta e gerência (exclusão, atualização e entrada) das informações.

Atualmente, a aplicação, a base de dados e as imagens estão centralizadas em um servidor de dados sediado na Embrapa Informática Agropecuária. Contudo, o mesmo está apto a referenciar imagens externas a este domínio. Para tanto, a imagem a ser disponibilizada deverá ser catalogada previamente e ter seu acesso liberado.

A seguir, são descritas, sucintamente, as etapas executadas pelo CGI Utilis no processo de consulta à base de dados:

1. Recebe o documento denominado metaformulário, o qual é formado de comandos HTML e tags especiais. As tags formam uma linguagem específica interpretada pelo CGI.

2. Interpreta os comandos de sua linguagem de programação (tags) presentes no metaformulário.

3. Ativa o sistema gerenciador de banco de dados por meio do driver ODBC.
4. Repassa ao SGBD as questões SQL que constam do metaformuário. O SGBD executa as atividades de seleção ou inserção nas bases.

5. Acessa os resultados de consulta repassados pelo SGBD.

6. Formata o resultado na forma de outro documento HTML, com os comandos interpretados que se encontram no metaformulário.

O desenvolvimento das bases foi realizado com o auxílio do software Apache 1.3b6*, que simula um servidor HyperText Transfer Protocol (HTTP) para a plataforma PC, os drivers Open DataBase Connectivity (ODBC), responsáveis pela padronização da comunicação entre sistemas diversos, e o Sistema Gerenciador de Banco de Dados (SGBD) utilizado foi o Microsoft Access 97.

\section{APÊNDICE}

\section{Orientações para a descrição do conteúdo dos elementos metadados}

\section{Título}

Orientação para a descrição do conteúdo - Na dúvida sobre o que constitui o título, incluir as variações em segunda e subseqüentes iterações. Se o recurso está em HTML, visualizar o documento-fonte e assegurar-se de que o título identificado no header da página está incluído também como um metatítulo (a menos que o elemento metadado DC esteja embutido no próprio documento). No caso de imagens que não possuam título, o catalogador do recurso deve atribuir um título breve e descritivo, transcrito entre colchetes

Exemplos:

Camu-camu

Cancro da haste da soja

Lagarta da soja

Catalog of rust types

[Biblioteca da Embrapa Informática Agropecuária] 


\section{Autor ou Criador}

Orientação para a descrição do conteúdo - Os nomes dos autores ou criadores devem ser indicados na mesma ordem que eles aparecem na publicação/recurso. Nomes pessoais devem ser indicados pelo sobrenome ou pelo primeiro nome de família, seguidos pelos prenomes. Na dúvida, atribuir o nome como aparece, e não invertê-lo. A autoria de fotografias e slides deve ser atribuída ao fotógrafo; se houver mais de um, indicar no elemento Colaborador.

\section{Exemplos:}

Carvalho, J.E.U. de

Yorinori, J.T.

Embrapa Arroz e Feijão

No caso de organizações em que existe claramente a presença de uma hierarquia, indique as partes dessa hierarquia da maior para menor, separadas por pontos.

Brasil. Ministério da Agricultura e do Abastecimento Embrapa. Diretoria Executiva (Brasília, DF)

NÃO:

Diretoria Executiva da Embrapa

Se não está clara a existência de hierarquia, ou se há dúvida sobre qual parte do corpo é maior ou menor, atribua o nome da forma como aparece no recurso.

Comitê Gestor Internet/Brasil

New York Botanical Garden Herbarium

Associação dos Engenheiros Agrônomos do Estado de São Paulo

Organização das Cooperativas Brasileiras

Se Criador e Publicador são o mesmo, não repetir o nome na área de Publicador. Se a natureza da responsabilidade é ambígua, a prática recomendada é usar nome de organizações para Publicador, e nome de pessoa para Criador. Em casos de menor responsabilidade na criação, atribuí-la no elemento Contribuidor.

\section{Palavras-chave}

Orientação para a descrição do conteúdo - Selecionar palavraschave do título ou do item descrição do recurso. Se o assunto do recurso é uma pessoa ou uma organização, usar a mesma forma do nome como se a pessoa ou organização fosse a Criadora; neste caso, não repetir o nome no elemento de Criador.
Em geral, escolher as palavras mais significativas para palavras-chave, evitando descrever um item em particular. Recomenda-se o uso de vocabulários controlados, como o Agrovoc, Thesagro.

\section{Exemplos:}

Cancro da haste da soja; Doenças da soja; Lagarta da soja; Camu-camu; Frutas da Amazônia

\section{Categoria}

Orientação para a descrição do conteúdo - Atribuir categorias de assunto, de acordo com código de classificação adotado. No caso do Rural Mídia, está sendo usado o AGRICOLA Subject Category Code, código usado pela National Agricultural Library-NAL.

\section{Exemplos:}

Pest of Plants (Insects and Other Arthropods) - [F821] Plant Production (Horticultural Crops) - [F110] Plant Diseases (Fungal) - [F831]

\section{Descrição}

Orientação para a descrição do conteúdo - Dado que o campo de Descrição é potencialmente uma fonte rica de vocabulário indexável, deve-se tomar cuidado para fornecer este elemento sempre que possível. Algumas coleções de metadados podem incluir descrição de conteúdo (análise espectral de um recurso visual, por exemplo), a qual não se encontra no recurso que está sendo descrito. Nesse caso, este campo poderia conter um link para o local onde se encontra a descrição do recurso.

Informação descritiva pode ser tomada do próprio recurso, caso não haja abstract ou outra descrição estruturada disponível. Se uma descrição não pode ser encontrada na introdução, páginas iniciais, primeiros parágrafos, deve-se fazer uma sondagem no provedor do metadado, para que se busquem elementos para a descrição do recurso. A descrição deve ser limitada a poucas e breves frases.

\section{Exemplos:}

Mancha vermelha na haste causada Cercospora kikuchii, de desenvolvimento superficial e freqüentemente confundido com cancro da haste;

Fruto de camu-camu. 


\section{Publicador}

Orientação para a descrição do conteúdo - A especificação deste campo visa a identificar a entidade que provê acesso ao recurso. Se o Criador e Publicador são o mesmo, não repetir o nome no campo Publicador. Se a natureza da responsabilidade é ambígua, recomenda-se usar Publicador para organizações (autores corporativos) e Criador para indivíduos (autores pessoais). Em caso de menor responsabilidade na criação, usar o campo Contribuidor.

Para imagens, dada sua natureza não-comercial de produção, o elemento Publicador nem sempre se aplica.

Exemplos:

Embrapa Trópico Úmido

Embrapa Soja

\section{Colaborador}

Orientação para a descrição do conteúdo - Aplica-se a mesma orientação recomendada para o elemento Criador na descrição de nomes de pessoas ou organizações.

\section{Data}

Orientações para a descrição do conteúdo - Se a data completa é desconhecida, mês e ano (YYYY-MM) ou só ano (YYYY) pode ser usado. Outras formas de descrição são passíveis de uso, porém podem não ser interpretadas facilmente por usuários ou software.

1998-02-16

1998-02

1998

No caso de recursos eletrônicos, cujos conteúdos originais são passíveis de modificações constantes, adotar a data em que o recurso está sendo cadastrado na base de dados.

\section{Tipo}

Orientação para a descrição do conteúdo - Este elemento deve descrever o gênero do conteúdo do recurso. Veja http://sunsite.berkeley.edu/Metadata/structuralist.html para conhecer mais sobre a aplicação deste elemento. Uma lista mínima é recomendada pelo Dublin Core:

\footnotetext{
- texto

- imagem

- som
}

- dados

- software

- interativo

- evento

- objeto físico

os quais são usados como segue:

Texto - Recursos que na maioria das vezes são de natureza textual, cujo conteúdo é formado de palavras para leitura, mas pode incluir imagens de texto, tabelas e outros formatos do gênero texto. Exemplos: abstracts, anúncios, correspondências (e-mail, cartas, cartões postais), dicionários, home page, índices, manuais, manuscritos, minutas, monografias, panfletos, poemas, pré-print, proceedings, publicações seriadas (periódicos, jornais), relatórios técnicos, teses.

Imagem - $\mathrm{O}$ conteúdo é principalmente representação visual simbólica em duas dimensões, e não é texto, tais como imagens e fotografias de objetos físicos, pinturas, impressões, desenhos, assim como outras imagens e gráficos, animações, diagramas, filmes, mapas, notação musical. Exemplos: imagem em movimento (animação, filme), fotografias, gráficos.

Som - O conteúdo é principalmente áudio, tais como música, discurso, sons gravados. Exemplos: som ambiente, efeitos sonoros, música, narração, discurso.

Dados - Informação codificada em listas, tabelas, bases de dados etc., necessariamente, em formato disponível para processamento imediato por computador, tais como planilhas eletrônicas, bases de dados, dados de GIS. Exemplos: dados numéricos, espaciais, espectrais, estatísticos e textos estruturados (incluindo dados bibliográficos e relatórios de bases de dados).

Software - Programa de computador em código-fonte ou compilado, binários executáveis, que podem estar disponíveis para instalação não transitoriamente em outra máquina. Podem estar subdivididos com o nome da linguagem de programação usada. Para software que existe somente para criar um ambiente interativo, usar o gênero "interativo".

Interativo - Recursos que requerem interação do usuário para serem entendidos, executados ou experimentados. Exemplo: estilos de páginas web, applets, objetos de aprendizagem multimídia. Um ambiente projetado para envolvimento interativo com um ou mais usuários. Exemplos: serviços de bate-papo, realidade virtual e jogos. 
Evento - Ocorrência não persistente baseada no tempo. Metadado para um evento fornece informação descritiva que é a base para a descoberta do propósito, local, duração, responsável e links e recursos relacionados ao evento. $\mathrm{O}$ recurso tipo evento pode não ser recuperável, se a instanciação descrita expirou ou ainda está acontecendo. Exemplos: exposição, web conferência, workshop, performance, open day, ensaios.

Objeto físico - Objetos ou substâncias tridimensionais, os quais não são necessariamente texto ou imagem. Exemplo: uma pessoa, um computador, uma grande pirâmide, uma escultura etc. Representações digitais ou substitutos desses objetos devem usar imagem, texto ou outros tipos.

Exemplos: texto, imagem, som, dados, software, interativo, evento, objeto físico.

\section{Formato}

Orientação para a descrição do conteúdo - Formatos, como texto/html, ASCII, arquivos Postscript, aplicações executáveis, imagem JPEG, podem ser incluídos neste campo. Indique um formato, consultando Internet Media Types (http://www.isi.edu/in-notes/iana/assignments/ media-types/media-types) (MIME types). A princípio, formatos podem incluir livros, publicações seriadas ou outras mídia não eletrônicas.

Exemplos:

gif

jpeg

$\mathrm{rtf}$

html

pdf

voice

mpeg

$\operatorname{rfc} 822$

multipart/voice-message

http

iges

ms-access

\section{Acesso}

Orientação para a descrição do conteúdo - Este elemento também pode ser usado por identificadores locais (por exemplo, números de ID ou números de chamada), indicados pelo Criador do recurso para um item/recurso em particular.
Exemplos:

http://purl.oclc.org/metadata/dublin_core/

http://www.agnic.org/agdb/catrust.html\# top_txt

03854247-28 [ISBN]

H-A-X 5690B" [um número qualquer atribuído pela editora]

\section{Identificador de recurso}

Orientação para a descrição do conteúdo - Atribuir uma string ou número que garanta a identificação, de forma única, do registro do recurso na base de dados.

Exemplos:

$1,2,3,4 \ldots ., 40$

IMG374-2

\section{Fonte}

Orientações para a descrição do conteúdo - Em geral, inclua nesta área informações que não se adequam facilmente ao elemento Relação.

Exemplo: 025.322 (no caso, trata-se do número de classificação para a versão impressa do recurso da qual a presente foi digitalizada, cujo exemplo pode ser visto em:

http://www.cnptia.embrapa.br/projetos/biblio/ orientacoes.pdf

\section{Idioma}

Orientações para a descrição do conteúdo - Informação textual sobre o idioma do recurso. Se o conteúdo está em mais de um idioma, indicá-los.

Exemplos:

inglês;

francês;

OU, inglês; francês, espanhol (quando o recurso estiver em várias idiomas)

OU, dinamarquês com abstract em francês.

\section{Relação}

Orientação para a descrição do conteúdo - Um identificador de um segundo recurso e seu relacionamento com o presente recurso. Este elemento permite fazer vínculos de recursos relacionados ao recurso que está sendo descrito. 
Exemplos incluem uma edição de um trabalho (IsVersionOf - Versão de), uma tradução de um trabalho (IsBasedOn- Baseado em), um capítulo de um livro (IsParteOf - Parte de), uma transformação mecânica de um conjunto de dados em uma imagem (IsFormatOf Formato de). Tipos de relacionamentos podem ser selecionados de uma lista preparada pelo Dublin Core Working Series.

Segue lista de tipos que contempla a maioria das relações:

Parte de (IsPartOf)

Possui parte (HasPart)

Versão de (IsVersionOf)

Possui versão (HasVersion)

Fomato de (IsFormatOf)

Possui formato (HasFormat)

Referências (References)

Referenciado por (IsReferencedBy)

Baseado em (IsBasedOn)

Base para (IsBasisFor)

Requer (Requeres)

Requerido por (IsRequiredBy)

A indicação da relação deve ser feita observando-se a lista acima.

Exemplos:

Relações parte/todo (part/whole) são aquelas nas quais um recurso é uma parte física ou lógica de outra.

Título: Gombrich's Story of Art

Relação: Possui/existe versão; 13th Edition, 1972

[Relações de versão são aquelas nas quais um recurso é uma edição histórica de outro recurso pelo mesmo criador]

Título: Candle in the wind

Data: 1997

Criador: John, Elton

Tipo: som

Descrição: Tributo à morte da Princesa Diana

Relação: Versão da canção de Elton John de 1976 "Candle in the wind"

Relações de transformação de formato são aquelas nas quais um recurso é derivado de outro por uma reprodução ou reformatação tecnológica que fundamentalmente não é uma interpretação, mas pretendeu ser uma representação.

Título: Electronic AACR2

Relação: Formato de "Anglo-American Cataloging Rules, 2nd edition"
Título: Landsat TM dataset of Arnhemland, NT, Australia

Relação: Possui formato arnhem.gif

Relações de referência são aquelas nas quais o autor de um recurso cita, reconhece, discute, ou, caso contrário, faz reivindicações sobre outro recurso.

Título: Morgan’s ancient society

Relação: Referenciado por Engels na obra "Origin of the Family, Private Property and the State"

Título: Nymphet mania

Relação: Referência a Adrian Lyne's 'Lolita'

Relações criativas são aquelas nas quais um recurso é uma performance, produção, derivação, adaptação ou interpretação de outro recurso.

Título: O romance de Peter Carey "Oscar and Lucinda”

Relação: Base para o filme de 1998 "Oscar and Lucinda"

Título: O filme "My Fair Lady"

Relação: Baseado na peça de Shaw "Pygmalion"

Relações de dependência são aquelas nas quais um recurso requer outro recurso para seu funcionamento, distribuição ou aprovação, e não pode ser usado sem o recurso relacionado estar presente.

Título: program.c

Relação: Requer stdio.h

Título: List of Internet Media Types

Relação: Requerida por Dublin Core Format element

\section{Cobertura}

Orientação para a descrição do conteúdo - Se este elemento é usado para informação espacial ou temporal, cuidar para fornecer dados consistentes que possam ser interpretados pelos usuários. Para a maioria das aplicações simples, em que nomes lugar ou datas de cobertura poderiam ser úteis, se a informação é numérica ou alfabética pode ser o suficiente para diferenciar. Para aplicações mais complexas, importância deve ser dada à qualificação adicional.

Exemplos:

Cobertura (temporal): 1995-1998

Cobertura (temporal): século XX

Cobertura (espacial - abrangência nacional): Brasil

Cobertura (espacial - abrangência em vários países): internacional

Cobertura (espacial): região amazônica

Cobertura (espacial): interior da Bahia 


\section{Direito autoral}

Orientações para a descrição do conteúdo - Até o presente, usado somente na forma de uma declaração, ou ainda pela indicação de uma URL à qual estará vinculada uma informação sobre direitos autorais.

\section{Exemplos:}

Direitos autorais reservados: Embrapa Informática Agropecuária

http://www.microsoft.com/Windows/windowsmedia/ features/DRM/default.asp

\section{Contato}

Orientação para a descrição do conteúdo - Este elemento deve ser usado para indicar nome pessoal, ou institucional, por meio do qual pode ser estabelecido contato com o Autor/Criador e/ou Publicador do recurso.

Exemplos:

e-mail:cnptia@cnptia.embrapa.br

Embrapa Informática Agropecuária. Área de Comunicação e Negócios - e-mail: acn@cnptia.embrapa.br

\section{BIBLIOGRAFIA}

1. CURTY, M.G.; SUGUINO, R. Base de dados para indexação de slides com a utilização do Microlsis. In: SEMINÁRIO NACIONAL DE BIBLIOTECAS UNIVERSITÁRIAS, 9., 1996, Curitiba, PR. A biblioteca universitária e a sociedade da informação: anais. [Curitiba]: UFPR, [1996]. Disquete 3 1/2"

2. DUBLIN CORE METADATA INITIATIVE. A user guide for simple Dublin Core: draft version 5.1. URL: http://purl.oclc.org/dc/ documents/working_drafts/wd-guide-current.htm Consultado em 21 jul. 1999

3. MURPHY, A.; ENSER, P. Accessing the visual heritage: metadata construction at the Science \& Society Picture Library. Vine, v.107, p.58-64, 1997.

4. MOURA, M.F.; OLIVEIRA, P.C. de. Aplicativo de consulta aos dados metereologicos do CNPH via internet: estimativas de medias e gráficos obtidos "online". Campinas: EMBRAPA-CNPTIA, 1998. 60p. (EMBRAPA-CNPTIA. Relatorio Tecnico, 5).

5. PAZIN, R.A., comp., org. Indexação de multimeios. 2.ed. Curitiba, Ed. da URPR, 1993. 50p. (Didática, 3).

6. PERNAMBUCO, O.S.; ROLDAN, F.M. Construção de um banco de imagens na área da saúde: algumas reflexões. In: SEMINÁRIO NACIONAL DE BIBLIOTECAS UNIVERSITÁRIAS, 9., 1996 Curitiba, PR. A biblioteca universitária e a sociedade da informação: anais. [Curitiba]: UFPR, [1996]. Disquete 3 1/2"

7. PEROTA, M.L.L.R., comp., org. Multimeios: seleção, aquisição, processamento, armazenagem, empréstimo. 4.ed.rev. Vitória: Ed. Edufes, 1997. 183p.

8. RIBEIRO, A.M. de C.M. AACR2: Anglo-american cataloguing rules, 2nd edition: descrição e pontos de acesso. Brasília: Ed. do Autor, 1995. 577p.

9. SOUZA, T.B. de; CATARINO, M.E.; SANTOS, P.C. dos. Metadados: catalogando dados na Internet. TransInformação, Campinas, v.9, n.2, maio/ago. 1997. Disponível: http:// www.puccamp.br/ biblio/tbsouza92.html Consultado em 12 jul 1999.

10. TURNER, T.P.; BRACKBILL, L. Rising to the topo: evaluating the use of the HTML META tag to improve retrieval of Worl Wide Web documents through Internet serach engines. Library Resources $\mathcal{E}$ Technical Services, v.42, n.4, p.258-271, 1999.

11. WEIBEL, S. The Dublin core: a simple content description model for electronic resources. Bulletin of the American Society for Information Science, p.9-11, Oct./Nov. 1997.

\footnotetext{
Agradecimentos

Os autores agradecem aos colegas Marcelo Stefanelli e Suzilei Francisca de Oliveira Carneiro, pela valiosa colaboração na etapa de implementação e teste da aplicação para descrição de metadados.
} 